\title{
Universal Impulse Noise Suppression Using Extended Efficient Nonparametric Switching Median Filter
}

\author{
M. H. Suid ${ }^{1}$,M. A. Ahmad ${ }^{1}$,M. I. F. M. Hanif ${ }^{2}$, M. Z. Tumari ${ }^{3}$ and M. S. Saealal $\left.\right|^{3}$ \\ ${ }^{1}$ Faculty of Electrical \& Electronic Engineering, Universiti Malaysia Pahang,26600 Pekan, Pahang, Malaysia. \\ ${ }^{2}$ SEGi University, No. 9 Jalan Teknologi, Taman Sains Selangor, Kota Damansara, PJU 5, 47810 Petaing Jaya, Selangor. \\ ${ }^{3}$ Faculty of Engineering Technology, Universiti Teknikal Malaysia Melaka, Hang Tuah Jaya, 76100 Durian Tunggal, Melaka, Malaysia.
}

\begin{abstract}
This paper presents a filtering algorithm called extended efficient nonparametric switching median (EENPSM) filter. The proposed filter is composed of a nonparametric easy to implement impulse noise detector and a recursive pixel restoration technique. Initially, the impulse detector classifies any possible impulsive noise pixels. Subsequently, the filtering phase replaces the detected noise pixels. In addition, the filtering phase employs fuzzy reasoning to deal with uncertainties present in local information. Contrary to the existing conventional filters that only focus on a particular impulse noise model, the EENPSM filter is capable of filtering all kinds of impulse noise (i.e. the random-valued and/or fixed-valued impulse noise models). Extensive qualitative and quantitative evaluations have shown that the EENPSM method performs better than some of the existing methods by giving better filtering performance.
\end{abstract}

Keywords: Image processing, impulse noise, digital image, noise filtering, nonparametric switching median filter.

\section{INTRODUCTION}

With the usage of multimedia material becoming more widespread from day to day, visual information from high quality digital images plays an important role in many daily life applications. Unfortunately, digital images are frequently subjected to the contamination of impulse noise due to the interferences generated during transmission, acquisition in noisy environment [1]. Luckily, due to the advancement in digital technologies the level of noise density in digital images has dropped significantly to the low contamination rate. Still, even at low densities, the occurrence of impulse noise can severely damage the information in the original image. Therefore, it is essential to remove impulse noise effect before carrying any subsequent image processing task (e.g. segmentation, object recognition, data compression etc.). Mostly, these subsequent processing steps are largely affected by the quality of the filtered image [2].

For this purpose, many filters have been proposed. The median filter for instance, is a well-known nonlinear filter for suppressing impulsive noise due to its effectiveness and high computational efficiency [3],[4]. Despite its effectiveness in smoothing noise, the median filter tends to blur fine details and often destroys edges due to its clumsy filtering property that treats all the pixels equally without considering whether or not it is noise-free pixel.

The aforementioned drawbacks have led to the development of various switching-based filters, e.g. the switching median (SWM) filters along with the centre weighted switching median (CWSWM) filter [5], the Laplacian switching median (LSM) filter [6], progressive switching median (PSM) filter [7] and the multi-state median (MSM) filter [8], etc. Basically, this class of filtering scheme works based on the impulse detection mechanism which uses a fixed size filtering window and predefined threshold value to differentiate between noise and noise-free pixels. With the noise detector, these filters are shown to be more effective in terms of the detail and edge preservation compared to the uniformly applied conventional median filters. However, one disadvantage is that the switching rule is typically based on a fixed threshold for locally obtained statistics. This approach in certain circumstances tends to yield problem of pixel's misclassification and fails to replace the noise pixels.

Of late, works in [9] has come out with a more flexible switching-based filter called efficient nonparametric switching median (ENPSM) filter, for detail-preserving restoration. The ENPSM filter is customary based on the combination of local variance threshold in the impulse noise detection module and recursive restoration technique in the pixel restoration module. Even though this method performs well, yet it only touched on the filtering of the random-valued (RV) impulse noise corrupted cases.

Thus, in this paper we take one step further by focusing on the detection and suppression of any type of impulse noise models. By using the same existing recursive filtering technique in the ENPSM filter, the proposed technique called extended efficient 
nonparametric switching median (EENPSM) filter is equipped with histogram-based impulse noise detector which is specifically designed for accurate fixed-valued (FV) impulse noise detection.

\section{IMPULSE NOISE MODEL}

In theory, impulse noise contamination amplitude could fall either within the image dynamic range (i.e. RV impulse) or out of the range (i.e. FV impulse) and usually only certain percentages of pixels are altered. For more detail, let $x(i, j)$ and $o(i, j)$ be the gray level of the noisy image and the original image at location $(i, j)$, respectively. Then, the impulse noise model with noise density $\rho$ can be defined as:

$$
x_{(i, j)}=\left\{\begin{array}{l}
n_{(i, j)}: \text { with probability } \rho \\
o_{(i, j)}: \text { with probability } 1-\rho
\end{array}\right.
$$

where $n(i, j)$ is the noise pixel value. The image is likely to be contaminated by the RV impulse noise when $n(i, j)$ is uniformly distributed within the image dynamic range, i.e. $n(i, j) \in[N \min , N \max ]$. On the other hand, the simplest impulse noise model is the FV impulse noise; where $n(i, j)$ is assumed to take the maximal and minimal intensities, i.e. $n(i, j) \in(N \min , N \max )$.

Although many researchers have paid special attention in obtaining a good filter for on a particular impulse noise model, this proposed technique takes one step further by focusing on the removal of RV and FV impulse noise all at once. In practical, identifying this scenario is more challenging compared to smoothing the $\mathrm{RV}$ noise or FV noise alone since it is closer to the realworld situations.

\section{EFFICIENT NONPARAMETRIC SWITCHING MEDIAN FILTER}

In this section, the conventional ENPSM filtering algorithm proposed in [9] will be briefly reviewed. Given a noisy image and initial filtering window $W(i, j)$ of size $(2 N+1) \times(2 N+1)$, the RV noise detection stages of ENPSM are described as follows:

Step 1: Sort all elements within $W(i, j)$ in ascending order and find the median pixel $m(i, j)$.

Step 2: Compute the absolute luminance differences $\mathrm{d}(i \pm k, j \pm l)$ between $m(i, j)$ and all pixels in $W(i, j)$; $d(i \pm k, j \pm l)=|x(i \pm k, j \pm l)-m(i, j)| ; \quad$ for $-N \leq k, l \leq N$

$\underline{\text { Step 3: }}$ : Rearrange each value obtained in $\mathrm{d}(i \pm k, j \pm l)$ and set the predefined threshold $T_{E N P S M}$ as; $T_{E N P S M}=\operatorname{med}\{d(i \pm k, j \pm l) \mid:-N \leq k, l \leq N\}$.

Step 4: Mark the locations of 'noise' pixels (i.e. $\mathrm{d}(i \pm k$,

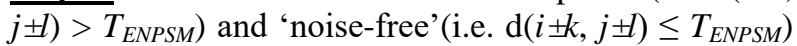
pixels in the binary noise map $M(i, j)$. Slide $W(i, j)$ to the next pixel and repeat Step 1 to Step 4 until the process is completed for the entire image.

This first part of this detection process is sufficient to handle impulse noise density (up to 20\%), largely for RV impulse noise. However, it is still not appropriate in the ENPSM to detect and replace the FV impulse noise accurately, particularly when the noise density is moderate or high.

\section{EXTENDED EFFICIENT NONPARAMETRIC SWITCHING MEDIAN FILTER}

The proposed filter is a modification of the original ENPSM filter, which operates on the same sliding window spatial filter that targets each pixel in a filtered image sequentially. The difference of the proposed filter with the former ones is that the proposed EENPSM filter modifies the existing ENPSM by adding one more process, in case when the case of FV contamination happens.

\subsection{Noise detection stage}

Taking the FV impulse noise into account, the noisy image histogram will be utilized by the proposed EENPSM filter. It is known that the peak intensities at the ends normally represent the FV noise intensities; e.g, see [10]. By employing the local maximum, the two fixed-valued impulsive intensities can be found by traversing the noisy image histogram from both ends and directed towards the centre of the histogram simultaneously. Once the local maximums denoted as $L_{M i n}$ and $L_{M a x}$, are found then the search will be stopped immediately. The detected local maximums represent the two fixed-valued FV impulse noise intensities. Furthermore, FV noise model is constructed based on the assumption that noise pixels will assume the two extreme values in the image dynamic range. Under some realworld situations, these FV noise pixels can be replaced by close approximations of their actual noise intensities [1113]. For example, pixels with the intensities value of 0 are possible to be replaced with 1 or 2 , and intensity value 255 will be replaced with 254 or 253 in an image stored as an 8-bit integer. Hence, distortion will be hardly detected by common FV impulse noise filters. At the end of the detection stage, a two-dimensional binary noise detection map $M(i, j)$ is formed based on:

$$
M(i, j)=\left\{\begin{array}{rr}
1, & {\left[d(i \pm k, j \pm l)>T_{\text {ENPSM }}\right]} \\
& \cap\left[x(i, j)=L_{\text {Min }} \cap L_{\text {Max }}\right] \\
0, & d(i \pm k, j \pm l) \leq T_{\text {ENPSM }}
\end{array}\right.
$$

where $M(i, j)$ in Step 4 is modified to produce a new version of $M(i, j)$ as shown in equation (2). Logic ' $1 \mathrm{~s}$ ' in the equation shows the positions of noisy pixels and logic ' 0 s' intended for those non-noisy ones.

\subsection{Noise filtering stage}

After binary noise mask $M(i, j)$ is formed, those pixels marked with $M(i, j)=1$ is then will be swapped by the estimated median value. Otherwise, the filtering action is 
skipped when $M(i, j)=0$ and the pixels will be left unprocessed. Once again, the proposed EENPSM algorithm uses a square filtering window $W_{\text {filter }}(i, j)$ with odd $(2 N+1) \times(2 N+1)$ dimensions and it is given as:

$$
\begin{array}{r}
W_{\text {filter }}(i, j)=\{x(i \pm k, j \pm l), \ldots, x(i, j), \ldots, x(i \pm k, j \pm l)\} \\
\text { where }-N_{\text {filter }} \leq k, l \leq N_{\text {filter }}
\end{array}
$$

For every ' $1 \mathrm{~s}$ ' pixel detected, the estimated median value is counted using only 'noise-free' pixels in the current filtering window. The calculation process is carried out using:

$$
\begin{array}{r}
m_{\text {est }}(i, j)=\operatorname{med}\{x(i \pm k, j \pm l), ., x(i, j), ., x(i \pm k, j \pm l)\} \\
\text { with } M(i \pm k, j \pm l)=0
\end{array}
$$

This criterion of choosing only 'noise-free' pixels is imposed to avoid selecting a 'noise' pixel as the estimated median pixel. Finally, the correction term to restore a detected noisy pixel is a linear combination between the current processing pixel and the estimated median pixel. The restoration term is given here as;

$$
y_{\text {EENPSM }}(i, j)=[1-M(i, j)] \square x(i, j)+M(i, j) \square m_{e s t}(i, j)
$$

\section{SIMULATION RESULTS AND DISCUSSIONS}

In this section, the practicability of the proposed EENPSM filter will be compared to the original ENPSM filter based on their simulation results. Three examples are provided to verify and justify the ideas described in Section 4 . In this experiment, the original $512 \times 512,8$ bits gray scale images Flower, Pens and Yacht were used in the simulation of the implemented filters. Those images are commonly used in image processing research and studies. Each of them was corrupted with fixed-valued and random-valued impulse noise ranging from $10 \%$ to $30 \%$. Both qualitative and quantitative assessments are employed to assess the performances of the proposed EENPSM and the original ENPSM filter. The quantitative assessment used here is the peak signal to noise ratio (PSNR) which is defined as:

$$
P S N R=10 \log _{10}\left(\frac{255^{2}}{M S E}\right) d B
$$

where MSE is the mean-squared error given as:

$$
M S E=\left(\frac{1}{M \times N}\right)^{M} \sum_{i=0}^{M-1} \sum_{j=0}^{N-1}[o(i, j)-y(i, j)]^{2}
$$

For the above mentioned formula, $M \times N$ is the image size with ' $M$ ' rows and ' $N$ ' columns, $y(i, j)$ is the filtered image and $o(i, j)$ is the original noise-free image.

As can be seen in Figure 1, at 10\% impulse noise density, the noise filtering performance of EENPSM filter is basically similar to the original ENPSM filtering algorithm. Both filters are found to be able of producing visible restored image at this noise level since the density of impulse noise is still low and not yet form any noise patches at this level

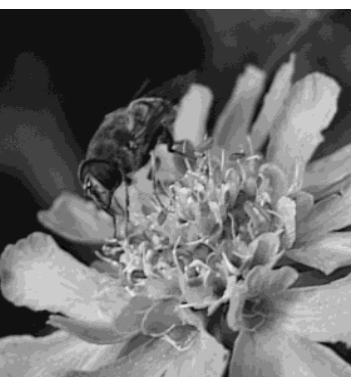

(a)

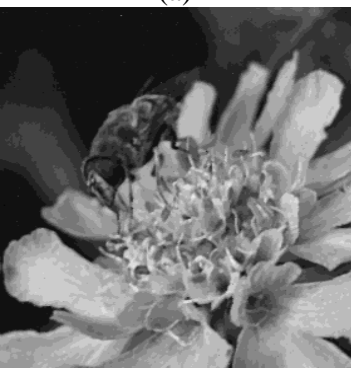

(c)

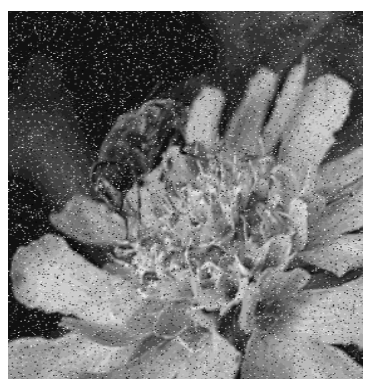

(b)

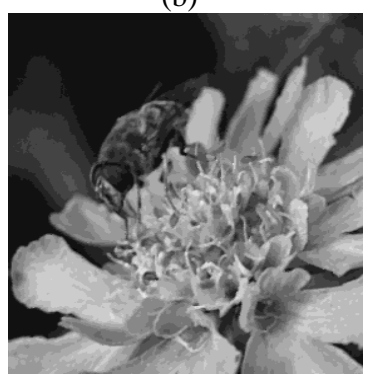

(d)
Figure 1. Simulation results on a portion of Flower with $10 \%$ density of impulse noise using; (a) original image, (b) noisy image, (c) ENPSM and (d) EENPSM.

However, in the Pens image (see Figure 2), we may notice that the proposed EENPSM filter gives better and clearer filtering result compared to the original ENPSM algorithm. The noise particles and effects are significantly reduced and at the same time the image details are well preserved. In contrast, we may be able to notice that some small noise spots are remained intact on the resultant images produced by the ENPSM filter. It is found that the original ENPSM filter has problem in the case of FV noise blotches (a place in image where a large number of fixed-valued impulse pixels may connect).

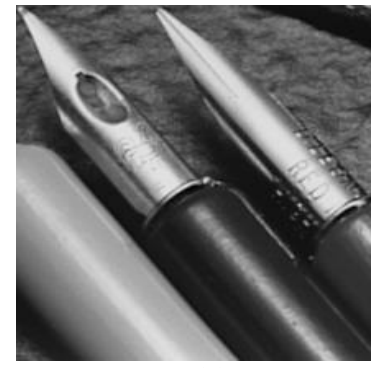

(a)

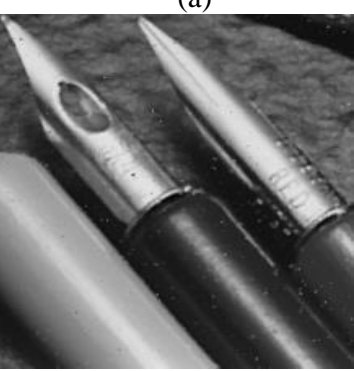

(c)

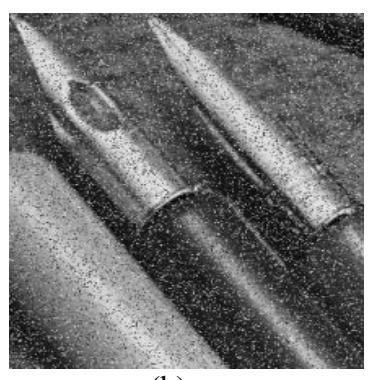

(b)

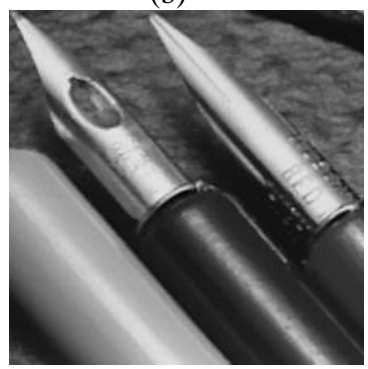

(d)
Figure 2. Simulation results on a portion of Pens with $20 \%$ density of impulse noise using; (a) original image, (b) noisy image, (c) ENPSM and (d) EENPSM. 
The similar phenomenons are obtained for the Yacht test image (shown in Figure 3), where the proposed EENPSM filtering algorithm consistantly outperforms the ENPSM filter by giving clearer image; even the density of noise in this image is increasing. This is due to the ability of the proposed algorithm to distinguish the FV noise pixels more dexterously as compared to the previous filtering version.
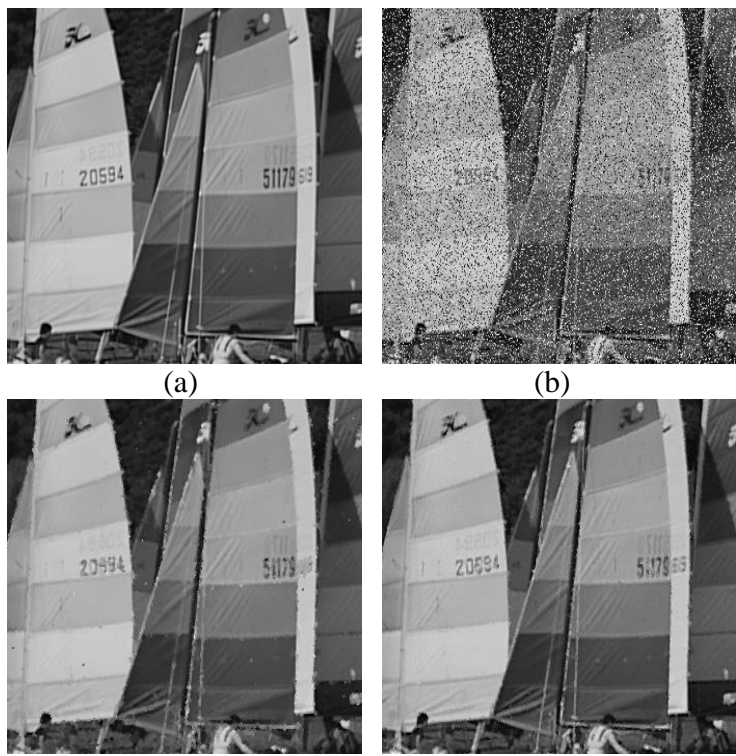

(c)

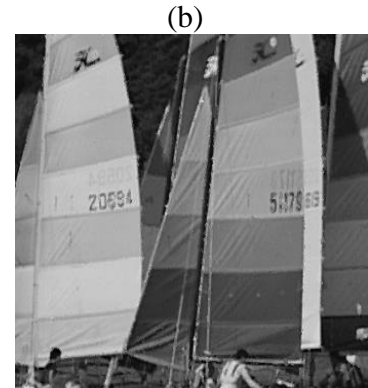

(d)

Figure 3. Simulation results on a portion of Yacht with $30 \%$ density of impulse noise using; (a) original image, (b) noisy image, (c) ENPSM and (d) EENPSM.

Table 1. Comparison of PSNR on Different Noise Level Restoration for Flower (Test Image)

\begin{tabular}{|c|c|c|c|}
\hline \multirow{2}{*}{ Algorithms } & \multicolumn{3}{|c|}{ PSNR (dB) } \\
\cline { 2 - 4 } & $10 \%$ & $20 \%$ & $30 \%$ \\
\hline ENPSM & 33.9590 & 31.9943 & 30.6433 \\
Proposed EENPSM & $\mathbf{3 4 . 5 3 0 8}$ & $\mathbf{3 3 . 2 5 9 4}$ & $\mathbf{3 1 . 6 1 8 5}$ \\
\hline
\end{tabular}

Table 2. Comparison of PSNR on Different Noise Level Restoration for Pens (Test Image)

\begin{tabular}{|c|c|c|c|}
\hline \multirow{2}{*}{ Algorithms } & \multicolumn{3}{|c|}{ PSNR (dB) } \\
\cline { 2 - 4 } & $10 \%$ & $20 \%$ & $30 \%$ \\
\hline ENPSM & 36.1737 & 33.5591 & 30.6339 \\
Proposed EENPSM & $\mathbf{3 7 . 5 0 1 1}$ & $\mathbf{3 4 . 8 1 2 3}$ & $\mathbf{3 2 . 8 4 5}$ \\
\hline
\end{tabular}

Table 3. Comparison of PSNR on Different Noise Level Restoration for Yacht (Test Image)

\begin{tabular}{|c|c|c|c|}
\hline \multirow{2}{*}{ Algorithms } & \multicolumn{3}{|c|}{ PSNR (dB) } \\
\cline { 2 - 4 } & $10 \%$ & $20 \%$ & $30 \%$ \\
\hline ENPSM & 31.5160 & 29.1585 & 26.6862 \\
Proposed EENPSM & $\mathbf{3 3 . 3 6 8 8}$ & $\mathbf{3 0 . 3 9 3 1}$ & $\mathbf{2 8 . 6 2 3 1}$ \\
\hline
\end{tabular}

Meanwhile, the numerical results for the used standard test images (i.e Flower, Pens and Yacht) are presented in Tables 1-3, respectively. Overall, it is made known that the proposed EENPSM unfailingly outperforms the previous algorithm at all level of universal impuse contamination case. It is evident that EENPSM filtering performance is tremendously consistent. In contrast, inconsistentce performances have been shown by the ENPSM filter with their PSNR values to have decreased intensely especially at the $30 \%$ noise level. Indirectly this result also shows that the conventional methods were unable to cater for the occurrences of noise in a proper manner especially when it comes to the fixed-valued contamination cases.

\section{CONCLUSIONS}

In this article, an extended version of ENPSM filter namely EENPSM for effective universal impulse noise restoration is presented. The variance thresholding and recursive pixel restoration techniques that involved in the design of the filter make it able to suppress both randomvalued and fixed-valued impulse noise effectively, at the same time preserving fine image edges and textures. Furthermore, this filter does not require any special tuning of parameter since its predefined threshold is established based on nonparametric framework. The simulation results indicate that a better noise filtering performance is achieved. In addition, the fuzzy reasoning could be embedded as part of its filtering mechanism, which permits us to exploit the effectiveness of fuzzy paradigm in handling imprecise local information. Overall, it is a feasible approach for removing the effects of low level universal impulse noise in digital images.

\section{Acknowledgement}

This work was partially supported by the Research and Innovation Department, University Malaysia Pahang and the Ministry of Higher Education under research grant RDU1703151.

\section{References}

1. X. Lan, Z. Zuo, Random-valued impulse noise removal by the adaptive switching median detects and detail preserving regularization, (2013).

2. K. K. V. Toh, H. Ibrahim and M. N. Mahyuddin. Cluster-based Adaptive Fuzzy Switching Median Filter for Universal Impulse Noise Reduction. IEEE trans. Consumer Electron., 56(4), 2560-2568 (2010).

3. J. Astola, P. Kuosmanen, Fundamentals of Nonlinear Digital Filtering, CRC, Boca Raton, FL, (1997).

4. R.C. Gonzalez, R.E. Woods, Digital Image Processing, Prentice Hall, New Jersey, (2002).

5. T. Sun and Y. Neuvo. Detail-Preserving Median Based Filters in Image Processing. Pattern Recognition Letters, 15 (1994) 341-347. 
6. S. Zhang and M. A. Karim. A new impulse detector for switching median filters. IEEE Signal Processing Letters, 9 (11), 360-363, (2002).

7. Z. Wang and D. Zhang. Progressive switching median filter for the removal of impulse noise from highly corrupted images. IEEE Trans. on Circuits and Systems II, 46 (1),78-80, (1999).

8. T. Chen and H. R. Wu. Adaptive impulse detection using centre-weighted median filter. IEEE Signal Processing Letters, 8 (1), 1-3 (2001).

9. M. H. Suid and N. A. M. Isa. Random valued impulse noise reduction in digital image using efficient nonparametric switching median filter. Scientific Research and Essays vol. 7(7), 813-823, (2012).

10. K. K. V. Toh and N. A. M. Isa "Noise Adaptive Fuzzy Switching Median Filter for Salt-and-Pepper Noise Reduction", IEEE Trans. Consumer Electron., vol. 17(3), 281-284, (2010).
11. N. I. Petrovic and V. Crnojevic, " Universal impulse noise filter based on genetic programming," IEEE Trans. Image Process., vol. (17)(7), 11091120, (2008).

12. M. Ismaeil, K. Pritamdas, K. J. K. Devi and S. Goyal, "Performance analysis of new adaptive decision based median filter on FPGA for impulsive noise filtering," 1st International Conference on Electronics, Materials Engineering and NanoTechnology (IEMENTech), Kolkata, 2017, pp. 1-5, (2017).

13. M. S. Darus, S. N. Sulaiman, I. S. Isa, Z. Hussain, N. M. Tahir and N. A. M. Isa, "Modified hybrid median filter for removal of low density randomvalued impulse noise in images," 6th IEEE International Conference on Control System, Computing and Engineering (ICCSCE), Batu Ferringhi, 2016, pp. 528-533, (2016). 